\title{
STUDIES ON RED BLOOD CELL DIAMETER
}

\section{The Relative Diameter of Immature (Reticulocytes) and Adult Red Blood Cells in Health and Anemia, Especialiy in Pernicious Anemia}

BY ELBERT LAPSLEY PERSONS

(From the Medical Service of the Collis P. Huntington Memorial Hospital of Harvard University and the Thorndike Memorial Laboratory of the Boston City Hospital)

(Received for publication April 15, 1929)

It has been shown (1) (2) (4) that curves of the frequency of the diameters of normal erythrocytes are symmetrical and uniform. In most cases of anemia classed as secondary, frequency curves of measurement of the red blood cell diameters are displaced to the left, or small side, of the normal and the mean diameter of the cells is less than normal. In contrast to this, in pernicious anemia in relapse the frequency curve usually is displaced to the right and the mean diameter is greater than normal. In marked remissions of pernicious anemia induced by the feeding of suitable amounts of liver or potent liver extracts diameter frequency curves often approach normal (3) (9).

The irregularities of the frequency curves of the diameters of erythrocytes from pernicious anemia has aroused interest and PriceJones (5) has suggested that in view of the apparent skewness of these curves there may be three elements of red blood cell formation in the bone marrow, the normal, giving the basic curve, together with the smaller cells (anemic) and larger (pernicious) cells which impart the skewness. It was suggested to me by Dr. George R. Minot that a study of the relative diameter of the young blood corpuscles (reticulated red blood cells, reticulocytes) to adult red blood cells might throw light on the nature of the skewness and that such observations upon normal red blood cells and those from various types of anemia might give information concerning blood formation. Heretofore no especial attention appears to have been given to the actual or compara- 
tive sizes of the reticulocytes under varying conditions. Hawes (6) in 1909 , described them as uniformly larger than non-reticulated cells. Minot and Lee (10) and Key (7) have noted the occurrence of small reticulocytes. Gram (8) writes that "vital-stainable corpuscles" are "relatively thick elements" and "may dominate the picture in periods of forced regeneration of simple anemias and in hemolytic jaundice." Knowledge concerning the growth of tissue cells has been obtained by determinations of the nuclear-cytoplasmic ratio and this favored the idea that a comparison of the size of the mature and immature erythrocytes might help to explain certain features of the formation of red blood corpuscles. Twenty-six observations of this sort have been made of the erythrocytes of 5 normal and 19 pathological bloods, particularly from cases of pernicious anemia, and this paper concerns the results obtained.

\section{METHOD}

Measurements of the red blood cell diameters were made from some specimens of capillary and some of venous blood, stained supravitally with brilliant cresyl blue between two thin glass coverslips. The blood was then stained with Wright's stain and the preparations mounted. The method of measuring the diameters was the same as that described in previous papers of this series (2) (3). To obtain adult red blood cell diameters 250 consecutive cells having distinct boundaries were measured. Adult red blood cells were considered to be those with no intracellular structures such as reticulum, HowellJolly bodies, Cabot ring bodies or refractile granules (Isaacs). The large, indistinct, pale red blood cells occurring in occasional fields of pernicious anemia blood were also omitted on the ground that they probably represent changes occurring after the cells are formed rather than abnormalities in blood formation. They are so rare that their inclusion would have changed nothing of importance in the data except the upper limit of red blood cell size. The occurrence of polychromatophilia may be taken to indicate improper staining by the method used. Hence to be as certain as possible that all reticulocytes were stained, no preparation containing polychromatophilic cells without reticulum was used for measurements. The 100 reticulocytes measured were found among or close to the adult red blood cells which had 
been measured, so that, irrespective of absolute values, the relative values for adult red blood cell and reticulocyte diameters should be of significance.

The absolute variations in the diameters of the red blood cells from venous and capillary blood were found to be very slight and no greater than those of the red blood cells from two different preparations of the same capillary blood taken at the same time. Furthermore, the distribution of red blood cell diameters and the relations of reticulocyte to adult red blood cell diameters were practically identical in the two types of blood.

The mean or average diameter was computed arithmetically from the original data. The data were plotted for study as frequency curves and also as summation-frequency curves on Whipple's arithmetic probability paper (see Fig. 2) which converts a frequency curve showing perfect adherence to the "law of probability" into a straight line. The numerical ratio between mean reticulocyte and mean adult red blood cell diameter was computed for each case and is included in the data presented.

\section{COMPARISON OF RETICULOCYTES AND ADULT RED BLOOD CELLS}

Figure 1 demonstrates by frequency curves the size relationships found to exist between the diameters of adult red blood cells and reticulocytes in normal bloods and in a case of pernicious anemia in relapse. A comparison of the curve for the percentage distribution of the diameters of 1250 adult red blood cells from five normal bloods with that for $\mathbf{5 0 0}$ reticulocytes from the same bloods indicates that the distribution of sizes is strikingly similar, but that the reticulocytes in normal bloods are uniformly somewhat larger than the adult red blood cells. Since normal bloods contain only about one per cent of reticulocytes this curve for adult red blood cells very nearly corresponds with comparable ones for all red blood cells of normal blood which have been published (1) (2) (4) (8).

The case of pernicious anemia recorded as case 18 , table 1 , for which data are charted in figure 1 had a red blood cell count of 1.8 million per cu. $\mathrm{mm}$. and a typical blood picture of that disease. The measurements are similar in all respects to those of the cells from the other nine pernicious anemia patients studied with low red blood cell 
counts. The frequency curve (fig. 1) for the diameters of the 250 adult red blood cells measured is similar to other diameter frequency curves which have been published (2) (3) (4) (5) for all cells in com-

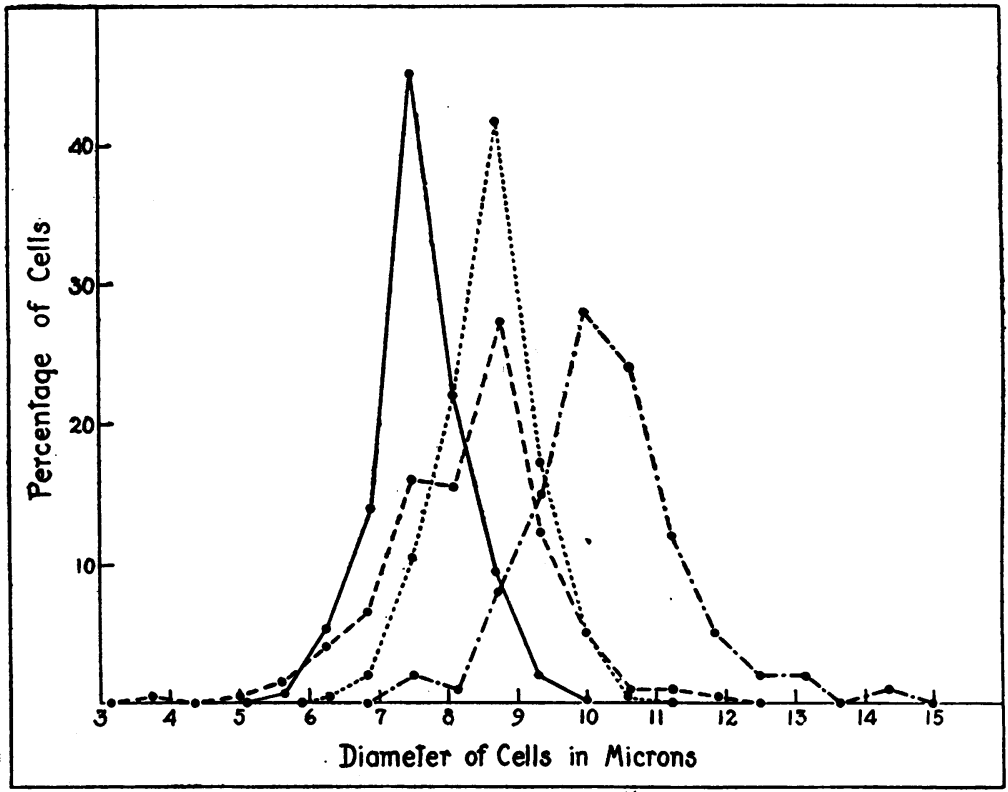

Fig. 1. Frequency Curves of the Size Relationships Between the Diameters of Reticulocytes and Adult Red Blood Celis in Normal Bloods and in a Case of Pernicious Anemia

The curves for normal bloods are constructed from the measurements of 1250 adult red blood cells and $\mathbf{5 0 0}$ reticulocytes from 5 individuals. The curves for the cells from a case (no. 18, table 1) of pernicious anemia are constructed from the measurement of 250 adult red blood cells and 100 reticulocytes.

- Normal adult red blood cells.

-..-.-.- Normal reticulocytes.

- - Pernicious anemia adult red blood cells.

- . - . Pernicious anemia reticulocytes.

parable cases of pernicious anemia. A comparison of this curve with that for 100 reticulocytes from the same blood shows a similarity of shape which, allowing for the smaller number of cells measured, is 
quite as striking as that between the diameter frequency curves for reticulocytes and adult red blood cells from normal bloods.

Table 1 shows the results of measurements of adult red blood cells and reticulocytes from 24 persons. These may be grouped in three classes, normal (5) "secondary" anemia due to various causes, (10) and pernicious anemia (9).

\section{A. Normal bloods}

Cases 1 to 5 were from healthy individuals who had normal blood pictures and the figures for their adult red blood cells conform fairly well to those obtained by other investigators for normal red blood cells. It is recognized that the adult red blood cell size of case 3 is lower than that of any normal blood which has been reported in this series of papers. The specimen was from a young adult male on whom repeated blood examinations have been made during the past four years without any abnormalities having been detected.

Normal reticulocytes average about 1 micron larger in diameter than normal adult red blood cells. The average of the means is 8.6 microns for the reticulocytes as against 7.6 microns for the adult red blood cells. The ratio of mean reticulocyte diameter to mean adult red blood cell diameter varies from 1.12 to 1.15 . The frequency curves obtained by plotting the averaged data from these 5 normal bloods are shown in figure 1. Summation-frequency curves of reticulocyte and adult red blood cell diameters from these bloods are so nearly straight that nothing but errors of measurement could be postulated to explain deviations and in each case the reticulocyte diameter distribution curve is almost parallel to that for adult red blood cell diameter.

\section{B. "Secondary" anemia}

In the cases of "secondary" anemia the reticulocyte-adult red blood cell diameter ratio (table 1 ) is lower than in the normal individuals.

The measurements marked $4 \mathrm{~b}$ and $5 \mathrm{~b}$ (table 1 ) were made on cells taken from normal individuals (cases 4 and 5) three days after the loss of from 500 to $600 \mathrm{cc}$. of blood, while measurements labelled $4 \mathrm{a}$ and $5 \mathrm{a}$ were made just before the blood was removed. In each case the red blood cells had fallen approximately half a million per cubic millimeter 


\begin{tabular}{|c|c|c|c|}
\hline \multirow{2}{*}{\multicolumn{2}{|c|}{ 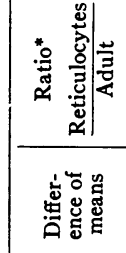 }} & & 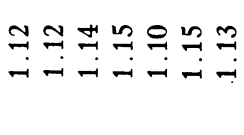 \\
\hline & & 莺 & 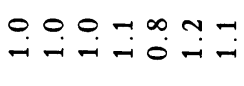 \\
\hline \multirow{4}{*}{ 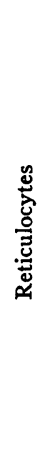 } & 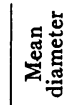 & 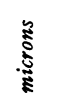 & 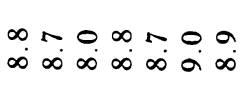 \\
\hline & 㤩 & 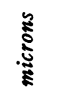 & 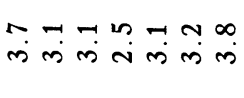 \\
\hline & 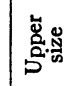 & $\stackrel{2}{:}$ & 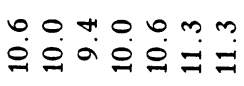 \\
\hline & : & 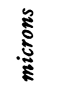 & $\begin{array}{l}a \\
0 \\
0\end{array}$ \\
\hline \multirow{6}{*}{ 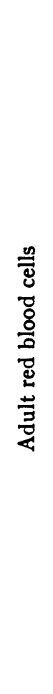 } & 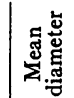 & 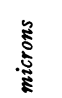 & 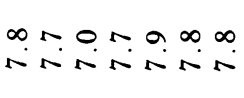 \\
\hline & 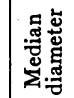 & 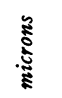 & 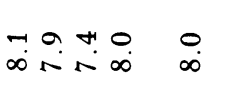 \\
\hline & 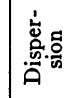 & 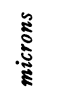 & そ๐ำ \\
\hline & $\begin{array}{l}\text { 范 } \\
\text { 点 }\end{array}$ & $\stackrel{\Sigma}{:}$ & $\vec{\forall} \tilde{m} \vec{m} \tilde{m} \dot{m}$ \\
\hline & 䓵: & 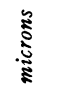 & 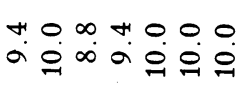 \\
\hline & 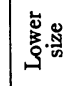 & 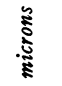 & i⿱ \\
\hline \multicolumn{2}{|c|}{ 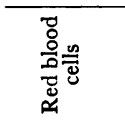 } & 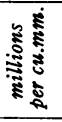 & 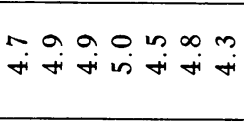 \\
\hline & & 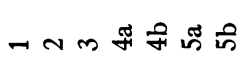 \\
\hline
\end{tabular}

\begin{tabular}{|c|c|c|c|}
\hline \multicolumn{2}{|r|}{ 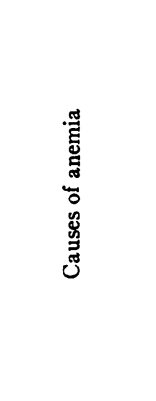 } & \multicolumn{2}{|r|}{ 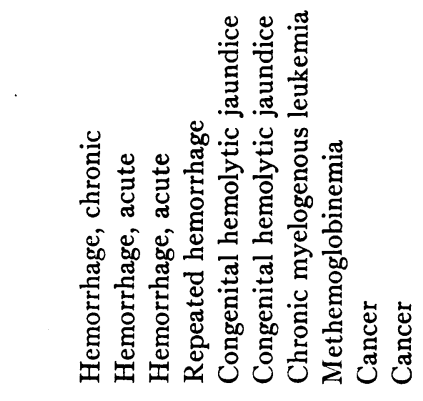 } \\
\hline \multicolumn{2}{|c|}{ 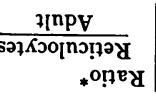 } & & 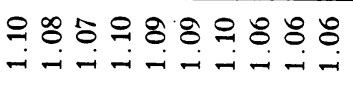 \\
\hline \multicolumn{2}{|c|}{ 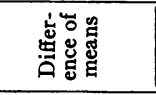 } & 资 & 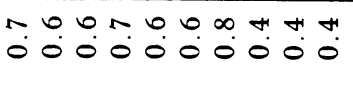 \\
\hline \multirow{4}{*}{ 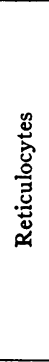 } & 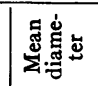 & 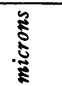 & 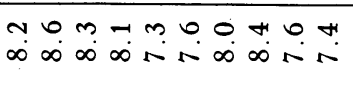 \\
\hline & $\begin{array}{l}\text { ङूँ } \\
\text { की }\end{array}$ & 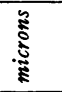 & 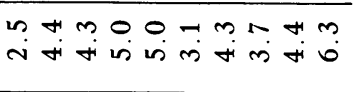 \\
\hline & 递: & 管 & 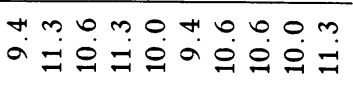 \\
\hline & 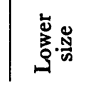 & 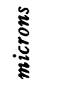 & 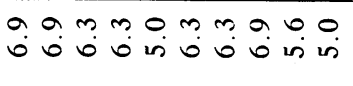 \\
\hline \multirow{4}{*}{ 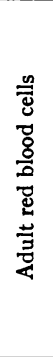 } & 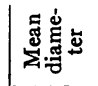 & 农 & 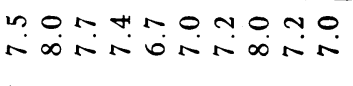 \\
\hline & 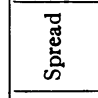 & $\frac{\pi}{\Sigma}$ & 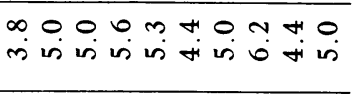 \\
\hline & 商: & 产 & 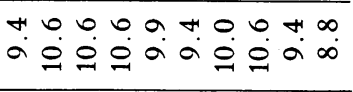 \\
\hline & 蒿: & 宸 & $\begin{array}{lllllll}0 & 0 & 0 & 0 & 0 & 0 & 0\end{array}$ \\
\hline \multicolumn{2}{|c|}{ 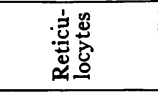 } & 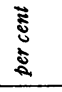 & 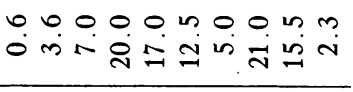 \\
\hline \multicolumn{2}{|c|}{ 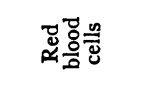 } & : & mîn \\
\hline \multicolumn{2}{|r|}{. } & & $0-\infty \circ 9$ \\
\hline
\end{tabular}




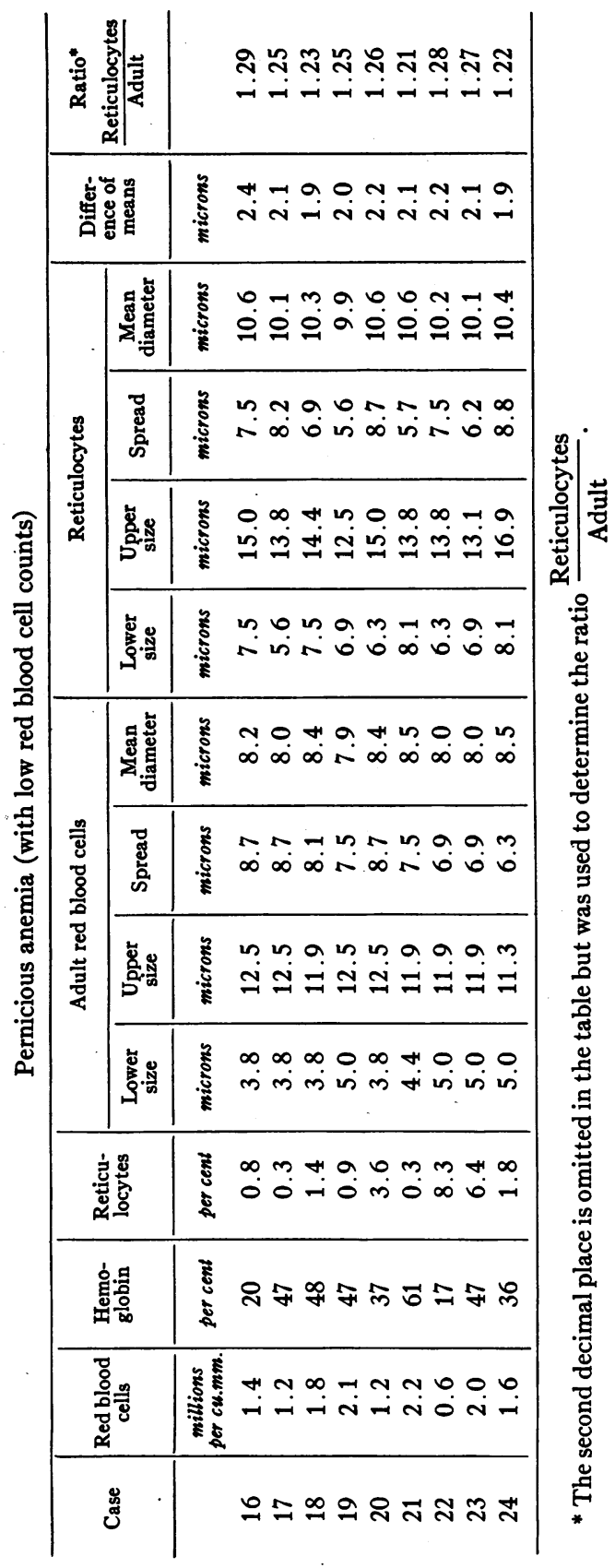


and about 4 per cent of reticulocytes were present indicating a response of the bone marrow. It is interesting that there is a slight increase in the adult red blood cell diameter in case 4 and no change for this value in case 5 , while in both cases the reticulocytes are smaller than before the loss of blood so that the reticulocyte adult red blood cell diameter ratio dropped from 1.15 to 1.10 in case 4 and from 1.15 to 1.13 in case 5. A larger series of similar cases must be studied before the importance of these changes can be properly evaluated.

The anemia in cases 6 to 9 was due to hemorrhage. Case 6 occurred in a woman who had had menstrual disturbances with abnormal blood loss and a slight anemia for several months before the specimen was taken. The mean adult red blood cell diameter while within normal limits, is smaller than that of most normal bloods, and the difference, 0.7 micron, between the mean reticulocyte and adult red blood cell diameters is definitely decreased, giving a reticulocyte-adult red blood cell diameter-ratio of 1.10. Cases 7 and 8 occurred in patients who had had a single severe hematemesis within a week of the time the specimen was taken. In these cases the mean adult red blood cell diameter is not abnormal, but the distribution has been changed, as is indicated by the increased value for the "spread." The spread of the reticulocytes is also somewhat increased. Again the differences between the mean reticulocyte and adult red blood cell diameters are reduced, so that the ratios between them are 1.08 and 1.07, but again the response of the bone marrow was slight. Case 9 occurred in a patient who had had repeated severe hematemeses during the five weeks previous to the day the specimen was taken, and while the "spread" of the adult red blood cells is definitely increased the mean adult red blood cell diameter is quite small. The "spread" of the reticulocyte diameters is also increased and the mean reticulocyte diameter is correspondingly reduced so that the reticulocyte-adult red blood cell diameter ratio is 1.10 , the same as that of the milder prolonged anemia due to blood loss (case 6).

Cases number 10 to 15 inclusive were of secondary anemia from causes other than hemorrhage. Case 10 and 11 were of congenital hemolytic jaundice and were both severe enough to justify splenectomy. The measurements of the cells from these two bloods are strikingly similar and resemble those reported by Whitcher (11); both retic- 
ulocyte and adult red blood cell "spreads" being increased and both sets of mean diameters being decreased considerably, so that the reticulocyte-adult red blood cell diameter ratio is 1.09.1

Case 12 was an example of "secondary" anemia accompanying chronic myelogenous leukemia and the measurements are very similar to those of case 9 with anemia from acute blood loss.

Case 13 exhibited varying degrees of anemia associated with proved methemoglobinemia existant for about eighteen months. At the time the specimen was taken the patient's red blood cell count was increasing and was about 2.5 million per cubic millimeter. The measurements of adult red blood cell diameters fail to show decreased mean cell size, but the "spread" is markedly increased. The many reticulocytes (21 per cent) are relatively small and the ratio of the mean diameters of the reticulocytes and adult red blood cells is low (1.06).

Case 14 showed a severe anemia due to cancer. The blood showed a high reticulocyte count, nucleated red blood cells and much poikilocytosis. The measurements show small adult red blood cells and reticulocytes and the ratio between their sizes is reduced to 1.06 .

Case 15 was the most interesting single case of the series. A blond Norwegian of $\mathbf{5 3}$ had severe anemia of about three months' duration. His history and physical examination suggested that he had pernicious anemia. He was transfused twice with blood and liver feeding commenced, but he died four days after the first transfusion. At autopsy the cause of death proved to be a scirrhous carcinoma of the stomach with metastases to the regional lymph nodes, liver and bone marrow. The measurements of the red blood cells after the first transfusion showed marked reduction in the mean adult red blood cell diameter and more than proportionate reduction in the mean reticulocyte diameter so that the ratio between them was reduced to 1.06. Until the time of death one of the chief findings which weighed against the diagnosis of pernicious anemia was these measurements of his red blood cell diameters.

Frequency curves plotted from measurements of the diameters of adult red blood cells in "secondary" anemia are similar to those

${ }^{1}$ An unpublished case of congenital hemolytic jaundice showing an increase in the mean diameter of the reticulocytes has been observed by Means and Thomas. 
obtained by other observers for all types of cells in similar bloods and the curves for reticulocyte diameters show corresponding shapes, but are not displaced as far to the right as are these curves for normal bloods. Summation-frequency curves on arithmetic probability paper are nearly straight for both reticulocyte and adult red blood cell diameters, if enough cells are measured to overcome the effect of the increased variation in size ("spread") found in "secondary" anemia bloods. Again the curve for reticulocyte diameter frequency is displaced to the right less than in normal bloods.

\section{Pernicious anemia}

Measurements from 9 cases of pernicious anemia with low red blood cell counts (cases 16-24, table 1) show that in every instance the mean red blood cell diameter is large, as is also the spread of both adult red blood cells and reticulocytes. The mean adult red blood cell diameter is close to normal in cases 19,22 and 23, but the mean reticulocyte diameter is distinctly above normal. Since the mean reticulocyte diameter is relatively very large, the ratios of the mean diameters are from 1.21 to 1.29 , or not less than 0.06 above the highest ratio obtained in a normal blood.

Frequency curves of adult red blood cell diameter measurements from these bloods are similar to those reported for pernicious anemia bloods by other observers (2) (3) (4) (5), and the reticulocyte diameter frequency curves correspond in shape to those of adult red blood cells from the same bloods, but are displaced much farther to the right than are those from normal bloods.

Summation-frequency curves plotted on arithmetic probability paper of 250 red blood cells from pernicious anemia bloods show deviations from the straight line plot for normal bloods, but in two cases in which 1000 cells of each were measured the resulting plots were as nearly straight as are corresponding curves from normal bloods.

Figure 2 shows the summation diameter frequency curve obtained from the measurement of 1000 adult red blood cells from case 20 , and a similar curve of diameter measurements of 100 reticulocytes from the same blood. These two curves are almost parallel and bear the same relation to each other that corresponding curves for red blood cells from normal bloods and secondary anemias do, except that the curve 
for reticulocyte diameters is displaced farther to the right. This very striking difference between the sizes of reticulocytes and adult red blood cells, which is demonstrated both by the figures and the curves for each of the nine cases of pernicious anemia with a low blood cell count and which is much less marked in all the other cases studied may be characteristic of the blood picture of this disease.

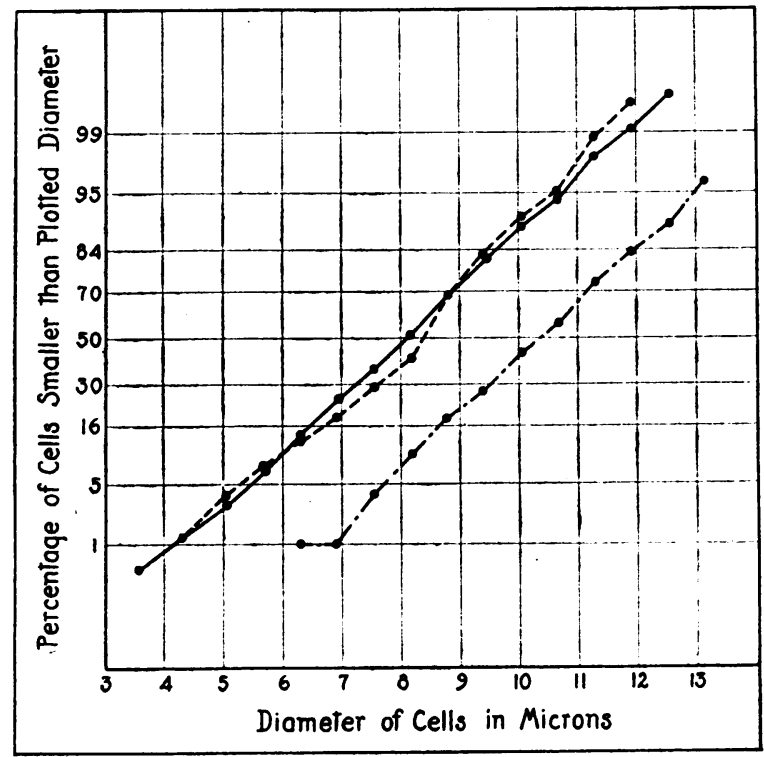

Fig. 2. Pernicious Anemia. Summation Diameter Frequency Curves of 1000 Adult Red Blood Cells, 250 Adult Red Blood Celis and 100 Reticulocytes from a Case of Pernicious Anemia Plotted on Arithmetic Probability PAPER

The curves for adult red blood cells show that the skewness of the curve based on only 250 cells disappears when a large number of cells (1000) are measured. 1000 adult red blood cells.

--250 adult red blood cells.

$-\cdot-\cdot-100$ reticulocytes.

\section{DISCUSSION}

The striking parallelism between the adult red blood cell and reticulocyte diameter distribution curves furnishes further evidence that the formation of reticulocytes is most intimately connected with 
the formation of adult red blood cells. In considering the relation of reticulocytes to adult red blood cells, there are two obvious possibilities. One is that the reticulocyte in the peripheral blood is a young red blood cell which will lose its reticulum in a few days, shrink somewhat in size, and become an adult cell. The other possibility is that reticulocytes are liberated from the marrow somewhat prematurely, and that they remain in the peripheral blood as such until dissolution takes place, but that the rate of their liberation is approximately proportionate to the rate of liberation of mature red blood cells. The present studies do not lead to deductions in favor of either of these hypotheses. The former is much the most probable one because one may observe extrusion and loss of reticular material in cells kept in plasma outside the body at $37.5^{\circ} \mathrm{C}$. The investigation does indicate that a close correlation exists between the sizes of reticulocytes and adult red blood cells in normal and pathological bloods.

The measurements from the twelve cases of "secondary" anemia recorded in table 1 indicate that when an extra load is thrown upon the bone marrow by "chronic" hemorrhage or red blood cell destruction it responds by throwing out reticulocytes which are relatively smaller than normal reticulocytes. (The actual measurements are smaller in only a fraction of the cases.) This is not what one would expect if the response of the bone marrow were simply one in which immature cells which would ordinarily remain in the marrow for a few days longer were liberated early, for studies of the bone marrow have shown that the red blood cells in it grow smaller as they mature.

In the four cases $(4 b, 5 b, 7$ and 8$)$ from which red blood cells obtained soon after hemorrhage were measured, it is reasonable to suppose that most of the adult red blood cells had been present before the hemorrhage occurred, so that there should be no marked abnormality in their measurements. In these bloods, however, the reticulocytes are relatively small which shows that the mean reticulocyte diameters tend to be low, while the mean adult red blood cell diameters remain at normal values.

In cases 6 and 9 one is justified in assuming that there had been time for some adjustment between the bone marrow and the circulating blood, or, in other words, that practically all of the adult red blood 
cells present in the blood had been formed after the hemorrhages, so that the parallelism between reticulocytes and adult red blood cells should be partially restored. That there was a trend in this direction is shown by the fact that the ratio between the mean diameters of reticulocytes and adult red blood cells is slightly higher than in the cases where there was not time for adjustment to take place. Although, under prolonged stimulus, the bone marrow was liberating smaller reticulocytes, the adult red blood cells were, by this time, smaller also.

From these and other data which have been published on the diameter of red blood cells in chronic "secondary" anemia it is apparent that the reaction of the bone marrow is to form red blood cells which are smaller than normal and which vary more in size as shown by the maximal and minimal measurements that yield the increased "spread." Jolly states that there is first a slight increase in mean diameter followed by the decrease observed in this study (12).

A comparison of the measure mens of three types of bloods, normal, anemia from loss of blood in which there has been no time for readjustment, and anemia from blood loss in which some readjustment must have taken place, shows that there is a somewhat delayed parallelism between the diameters of reticulocytes and adult red blood cells. It would necessarily follow that, in a patient who has had a single large hemorrhage, there will be a period in which the diameter ratio between reticulocytes and adult red blood cells would be less than that in normal bloods. Once the factor which stimulates the bone marrow to increased production is removed it should begin to form normal blood, including reticulocytes of normal size.

Other observers have reported irregularities in summation-frequency curves of the diameters of red blood cells from cases of pernicious anemia (5) (2). Measurements of 1000 red blood cells from each of two cases of pernicious anemia reported above resulted in curves which conformed to the "Law of Probability" as well as did curves for the diameter of red blood cells from normal bloods. Although the skewness of the curves obtained from measuring 250 red blood cells from the same cases was not marked, the result of measuring the larger number of red blood cells indicates that not all summationfrequency curves of the diameters of red blood cells from pernicious anemia bloods are skew curves (see fig. 2). 
There is no obvious explanation for the apparently constant increase in the relative diameter of reticulocytes in cases of pernicious anemia having low red blood cell counts. This increase is demonstrable in the diameter measurements, in the values for the ratio between mean reticulocyte and adult red blood cell diameters and is illustrated wel] by both frequency and summation-frequency curves plotted from the data. It is interesting to note that in the two cases of anemia due to congenital hemolytic jaundice reported above the mean reticulocyte diameter was relatively decreased. There is an excess of blood pigments in the plasma in this disorder as also occurs in pernicious anemia in relapse where the mean reticulocyte diameter is relatively increased. In the congenital disorder the red blood cell diameter measurements were similar to those found in "secondary" anemia, with no increase pigments in the plasma; hence, it is not likely that the presence of pigment has any relation to the diameter of the red blood cells.

\section{CONCLUSIONS}

1. There is a very intimate relationship between the sizes of reticulocytes (immature) and adult red blood cells in the same blood, as indicated by the fact that the shapes of the cell diameter distribution curves are almost identical.

2. Reticulocytes of normal bloods average enough larger than adult red blood cells of the same bloods so that the ratio of mean reticulocyte diameter to mean adult red blood cell diameter varies from about 1.12 to 1.15 .

3. In chronic "secondary" anemias the reticulocytes are relatively smaller than those from normal bloods, so that this ratio varies from 1.06 to 1.10 .

4. In cases of pernicious anemia having low red blood cell counts $(600,000$ to $2,200,000$ per cubic millimeter $)$ the reticulocytes are much larger than the adult red blood cells, so that the ratio between the mean diameters is between 1.21 and 1.29. This may be characteristic of the blood picture of this disease.

The author wishes to express his appreciation to Dr. George R. Minot for his direction of this work, and to Dr. J. H. Means and F. K. Thomas for their helpful criticism. 


\section{BIBLIOGRAPHY}

1. Price-Jones, C., Brit. Med. J., 1910, ii, 1418. The Variation in the Sizes of Red Blood Cells.

2. Bell, J. R., Thomas, F. K., and Means, J. H., J. Clin. Invest., 1926, iii, 229. Studies on Red Cell Diameter. I. In Health and in Pernicious Anemia.

3. Medearis, D. N., and Minot, G. R., J. Clin. Invest., 1927, iii, 541. Studies on Red Blood Cell Diameter. II. In Pernicious Anemia, before and during Marked Remission, and in Myelogenous Leukemia.

4. Grosh, L. C., and Stifel, J. L., Arch. Int. Med., 1925, xxxvi, 874. The Diameter of the Red Blood Cells in the Differentiation of Anemias.

5. Price-Jones, C., J. Path. Bact., 1922, xxv, 487. The Diameters of Red Cells in Pernicious Anemia and in Anemia Following Hemorrhage.

6. Hawes, J. B., Boston Med. and Surg. J., 1909, clxi, 493. A Study of the Reticulated Red Blood Corpuscle by Means of Vital Staining Methods. Its Relation to Polychromatophilia and Stippling.

7. Key, J. A., Arch. Int. Med., 1921, xxviii, 511. Studies on Erythrocytes with Special Reference to Reticulum, Polychromatophilia and Mitochondria.

8. Gram, H. C., Acta Med. Scandinav., 1927, lxvi, 295. On the Size and Form of the Red Cells in Normal and Anemic Cases.

9. Pijper, A., J. Med. Assoc. South Africa, 1928, ii, 483. Liver Diet and the Red Cell Diameter in Addisonian (Pernicious) Anemia.

10. Minot, G. R., and Lee, R. I., Boston Med. and Surg. J., 1917, clxxvii, 761. Treatment of Pernicious Anemia especially by Transfusion and Splenectomy.

11. Whitcher, B. R., Am. J. Med. Sci., 1925, clxx, 678. Microcytosis in Hemolytic Icterus.

12. Jolly, J., Traité Technique d'Hématologie. Vol. II, pp. 884-885. A. Maloine et fils. Paris, 1923. 